\begin{tabular}{|c|l|}
\hline Title & Magnetic clusters and fold energies \\
\hline Author(s) & Giga, Y oshikazu; Kubo, Motohiko; Tonegawa, Y oshihiro \\
\hline Citation & Hokkaido University Preprint Series in Mathematics, 666, 1-22 \\
\hline Issue Date & 2004 \\
\hline DOI & 10.14943/83817 \\
\hline Doc URL & http://hdl.handle.net/2115/69471 \\
\hline Type & bulletin (article) \\
\hline File Information & pre666.pdf \\
\hline
\end{tabular}

Instructions for use 


\title{
Magnetic clusters and fold energies
}

\author{
Yoshikazu Giga, Motohiko Kubo and Yoshihiro Tonegawa
}

Department of Mathematics, Hokkaido University, Sapporo 060-0810, Japan

\begin{abstract}
We are concerned with variational properties of a fold energy for a unit, dilation-invariant gradient field (called a cluster) in the unit disk. We show that boundedness of a fold energy implies $L^{1}$-compactness of clusters. We also show that a fold energy is $L^{1}$-lower semicontinuous. We characterize absolute minimizers. We also give a sequence of stationary states and discuss its stability. Surprisingly, the stability depends upon $q$, the power of modulus of the jump discontinuities, in the definition of the fold energy.
\end{abstract}

\section{Introduction}

H. A. M. van den Berg [8] studies magnetic thin film by proposing a model described as follows. A magnetic field $M$ on a domain $\Omega$ of $\mathbb{R}^{2}$ is called a magnetic cluster if $M \in L^{\infty}(\Omega)$ satisfies

$$
\begin{gathered}
\operatorname{div} M=0 \text { in } \Omega, \\
|M|=C \text { in } \Omega, \\
M \cdot \vec{n}=0 \text { on } \partial \Omega,
\end{gathered}
$$

where $C$ is a given constant and $\vec{n}$ is the unit outer vecter on $\partial \Omega$. He provided many examples of magnetic clusters. We use a 'fold energy' to characterize several important magnetic clusters and study its variational properties. For example, we study $L^{1}$-compactness and the stability of dilation-invariant clusters with respect to the fold energy defined formally by the form

$$
E^{q}(\nabla u)=\int_{\Sigma_{\nabla u}}|[\nabla u]|^{q} d \mathcal{S} .
$$

Here $M=(\nabla u)^{\perp}$ is a cluster, $q$ is a given positive number and $d \mathcal{S}$ denotes the line element. Roughly speaking, $\Sigma_{\nabla u}$ denotes the jump discontinuities of $\nabla u$ and $[\nabla u]$ represents the jump on $\Sigma_{\nabla u}$. This fold energy is formally an asymptotic limit of the Ginzburg-Landau type energy

$$
\int_{\Omega} \varepsilon^{-1}\left(1-|\nabla u|^{2}\right)^{q-1}+\varepsilon|\nabla \nabla u|^{2} .
$$


Closely related topics include [1], [2], [3], [5]. In general case without dilation invariance, we do not know the existence of global minimizers of the fold energy on the set of solutions of the 2-dimensional eikonal equation under suitable boundary conditions. There are examples showing that the distance function from $\partial \Omega$ is not a minimizer of the fold energy [2], [7]. The papers [1] and [5] establish compactness if $q=3$. We conjecture that compactness does not hold if $q>3$ [1], [5]. The paper [1] also showed that the fold energy is not lower semicontinuous for $q>3$ while Aviles and Giga [3] proved a lower semicontinuity result for the fold energy for $q=3$.

In this paper we are concerned with variational properties for a fold energy in the unit disk for a unit gradient field when a cluster is dilation invariant. To simply the wording from now on we call a unit, dilation-invariant gradient field a cluster. Our main results are

(1) $L^{1}$-compactness summarized as follows. If $\left\{E^{q}\left(\nabla u_{j}\right)\right\}_{j \in \mathbb{N}}$ is bounded, then there is an $L^{1}$-convergent subsequence of $\left\{\nabla u_{j}\right\}_{j \in \mathbb{N}}$. (Theorem 3)

(2) $L^{1}$-lower semi-continuity of $E^{q}$ with respect to $\nabla u$.(Lemma 2.8, Lemma 4.1) Our compactness and lower semi-continuity results do not depend on a positive number $q$. These results are significantly different from the case including general configuration as mentioned in a previous paragraph.

(3) Characterization of absolute minimizers such that $E^{q}(\nabla u)=0$.(Theorem 5.1)

(4)Stability of 'saddle point' type stationary configurations is studied.(Theorem 5.3)

We have given examples of stationary state whose stability depends on certain positive number $q$. There is a tendency that more configurations are stable for larger $q$. We conjecture that our examples of local minimizers exhaust all local minimizers but we did not touch this problem here.

We first define a fold energy for a finite wall cluster and consider its lower semicontinuous envelope for a limit cluster which is approximated by finite wall clusters. We show a lower semicontinuity of a fold energy for a finite wall cluster by characterizing a cluster by its argument function. This implies that the lower semicontinuous envelope is the same as original fold energy in a space of finite wall clusters. Unfortunately, we do not have an explicit representation of the envelope for a general limit cluster.

The compactness is also proved by studying argument functions of a finite wall clusters. Detailed study of argument functions also characterizes absolute minimizers. To show stability of saddle point like stationary clusters we reduce a problem in a space of finite wall clusters with the same number of walls and calculate the Hessian of the energy at that cluster. Fortunately, this provides stability results. 
Our space of limit clusters are not included in the space of functions of bounded variation (Lemma 3.2). Our example also shows that the Aviles-Giga space [1] is strictly larger than the space of functions of bounded variation, another example (non-cluster) is provided in [1]. For a seemingly smaller space than Aviles-Giga space but strictly large than the space of functions of bounded variation the notion of trace at jump discontinuities are extended by a recent paper of C. De Lellis and F. Otto [4].

Our characterizetion of global minimizers says that global minimizers are either a constant cluster or 'free cluster' (in the sense) that $\arg \nabla u(x)=\arg x$ or $\arg$ $\nabla u(x)=\arg (-x)$. Similar results (among all configurations) are proved by P.-E. Jabin, F. Otto and B. Perthame [7] for zero-limit $(\varepsilon \rightarrow 0)$ state of the GinzburgLandau energy given in the begining of the introduction.

The authors are grateful to Professor Robert Kohn for informative remarks.

This work was partly supported by the Grant-in-Aid for formation of COE 'Mathematics of Nonlinear Structures via Singularities'. The work of the first author was partly supported by the Grant-in-Aid for Scientific Research, No.14204011, No.1563408, the Japan Society for the Promotion of Science. The work of the third author was partly supported by the Grant-in-Aid for Scientific Research, No.14702001.

\section{Limit clusters and its fold energy}

To state our results precisely we prepare several spaces and notations. Let $B_{1}$ be the unit open disk centered at the origin in $\mathbb{R}^{2}$.

2.1. Clusters. We shall introduce a notion of a cluster and show that all cluster has a potential.

Definition 2.1. We call a vector field $V$ on $B_{1}$ a cluster if $V \in L^{\infty}\left(B_{1}\right)$ satisfies

$$
\begin{gathered}
\text { curl } V=0 \text { in } B_{1} \backslash\{0\} \\
|V|=1 \text { in } B_{1} \\
V(x)=V\left(x^{\prime}\right) \text { if } \arg x=\arg x^{\prime} .
\end{gathered}
$$

Let $K$ denote the set of all clusters. 


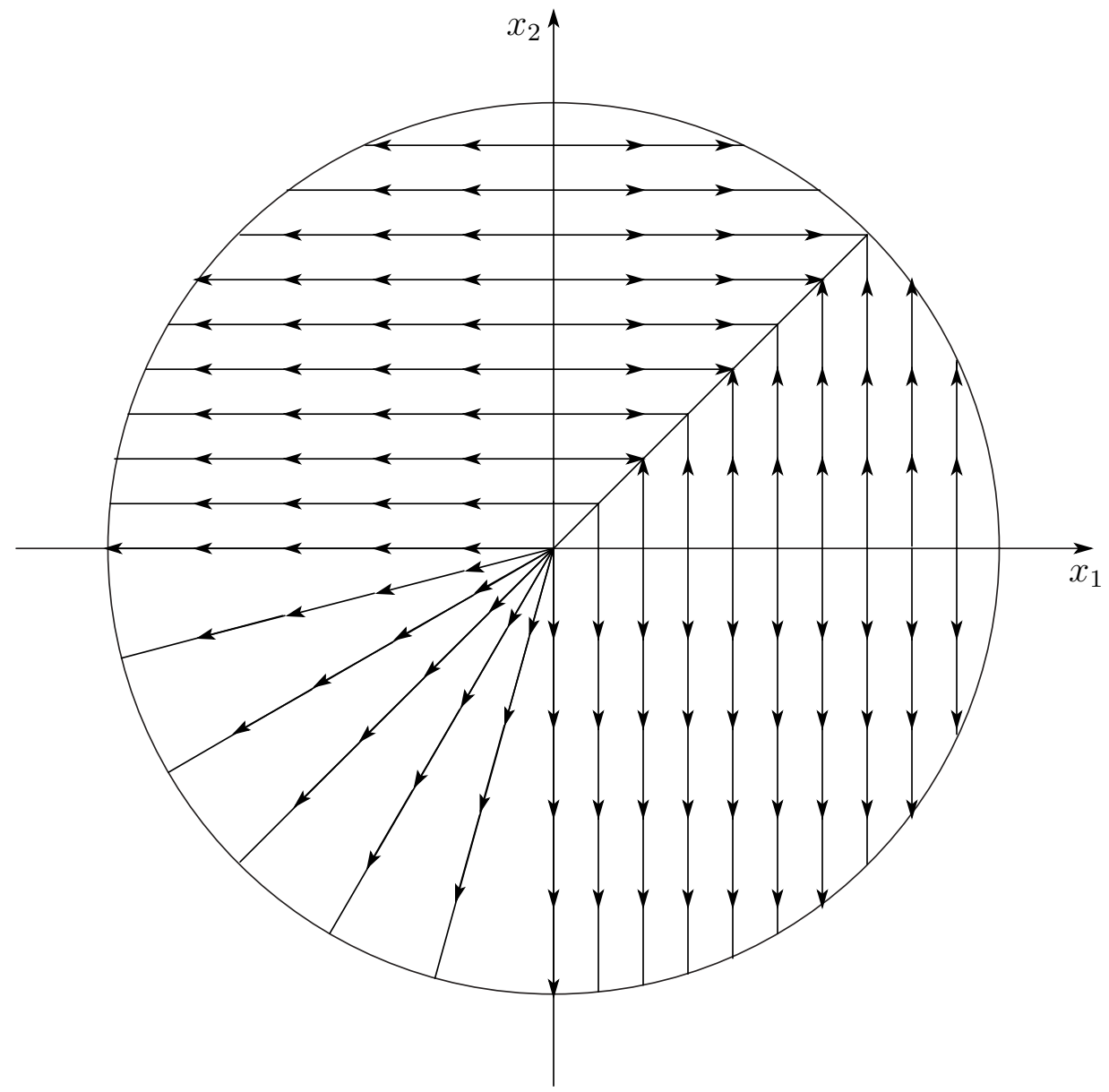

Figure 1. One of the examples of clusters.

Lemma 2.2. For any cluster $V$ there exists a function $u \in W^{1, \infty}\left(B_{1}\right)$ with $\nabla u=V$ and $\frac{u(x)}{|x|}$ depends only on $\arg x$. Here, $\nabla$ denotes the gradient operator.

Proof. By the definition of $V \in K$ there exists $g \in L_{l o c}^{1}(\mathbb{R})$ such that

$$
g(\theta+2 \pi) \equiv g(\theta) \text { modulo } 2 \pi
$$

and

$$
\arg V(x)=g(\arg x) \text { for } x \in B_{1} \text {. }
$$

We set

$$
a(\theta) \equiv \cos (g(\theta)-\theta) \text { for } \theta \in(0,2 \pi] .
$$

Since $\operatorname{curl} V=0$, we have that

$$
\cos \theta \frac{\partial}{\partial \theta}(\cos g(\theta))+\sin \theta \frac{\partial}{\partial \theta}(\sin g(\theta))=0,
$$

where $x=\left(x_{1}, x_{2}\right) \in \mathbb{R}^{2}, x_{1}=r \cos \theta, x_{2}=r \sin \theta$. Since

$$
\frac{\partial}{\partial \theta}(\cos (g(\theta)-\theta))=\frac{\partial}{\partial \theta}(\cos g(\theta) \cos \theta+\sin g(\theta) \sin \theta)
$$




$$
\begin{aligned}
= & \cos \theta \frac{\partial}{\partial \theta}(\cos g(\theta))+\sin \theta \frac{\partial}{\partial \theta}(\sin g(\theta)) \\
& +\sin g(\theta) \cos \theta-\cos g(\theta) \sin \theta \\
= & \sin g(\theta) \cos \theta-\cos g(\theta) \sin \theta \\
= & \sin (g(\theta)-\theta),
\end{aligned}
$$

we conclude

$$
a(\theta) \cos \theta-\frac{\partial a(\theta)}{\partial \theta} \sin \theta=\cos g(\theta)
$$

and

$$
a(\theta) \sin \theta+\frac{\partial a(\theta)}{\partial \theta} \cos \theta=\sin g(\theta)
$$

So if we take

$$
u(x)=|x| a(\arg x)
$$

then $V=\nabla u$.

2.2. Finite wall clusters. In this section we shall introduce a notion of a finite wall cluster and discuss its properties. The set of all finite wall clusters are denoted by $K_{f}(\subset K)$. We shall define a $q$-fold energy $E_{f}^{q}$ on $K_{f}$ and prove that $E_{f}^{q}$ is $L^{1}$-lower semicontinuous in $K_{f}$.

Definition 2.3. $\quad$ For a cluster $\nabla u$ let $\tilde{\Sigma}_{\nabla u}$ be the set of all points in $B_{1}$ where $\nabla u$ is not smooth.

By the definition a cluster $\nabla u$ satisfies $\nabla u(x)=\nabla u\left(x^{\prime}\right)$ for $\arg x=\arg x^{\prime}$. So there exists a unique set $\tilde{J}_{\nabla u} \subset(0,2 \pi]$ such that $\tilde{\Sigma}_{\nabla u}=\cup_{\theta \in \tilde{J}_{\nabla u}} l(\theta)$, where $l(\theta) \equiv\{x \in$ $\left.B_{1} \mid \arg x=\theta\right\}$. We call such $l(\theta)$ a wall of $\nabla u$.

Definition 2.4. We call a cluster $\nabla u$ a finite wall cluster if $\nabla u$ has finitely many walls. We denote the set of finite wall clusters by $K_{f}$. In particular, we denote the set of finite wall clusters which has no walls by $K_{f}^{0}$.

Notice that the cluster in figure 1 is one of finite wall clusters. This cluster has five walls $l\left(\frac{\pi}{4}\right), l\left(\frac{\pi}{2}\right), l(\pi), l\left(\frac{3}{2} \pi\right)$ and $l(2 \pi)$. By the definition of cluster $\nabla u$ there exists a function $g$ such that

$$
\nabla u(x)=(\cos g(\arg x), \sin g(\arg x))
$$

We call such a function $g$ an argument function of a cluster. Next we shall give several properties of argument functions of a finite wall cluster. 
Definition 2.5. Let $g$ be a function on $[0,2 \pi]$ such that $g \in C^{\infty}\left((0,2 \pi) \backslash \bigcup_{j=1}^{\alpha} \theta_{j}\right)$ with finitely many $\left\{\theta_{j}\right\}_{j=1}^{\alpha} \subset(0,2 \pi)$. We say $g \in Y$ if $g$ satisfies the following $\left(A_{1}\right)$ $\left(A_{4}\right)$.

$\left(A_{1}\right) \theta-\pi<g(\theta) \leq \theta+\pi$ for $\theta \in[0,2 \pi]$,

$\left(A_{2}\right) g(0+0)+2 \pi=g(2 \pi-0)$ or $\frac{|g(0+0)+2 \pi-g(2 \pi-0)|}{2}=2 \pi$,

where $g(\theta \pm 0) \equiv \lim _{\varepsilon \rightarrow 0+0} g(\theta \pm \varepsilon)$.

$\left(A_{3}\right) g \equiv C$ or $g(\theta) \equiv \theta$ or $g(\theta) \equiv \theta+\pi$ on $\left(\theta_{j}, \theta_{j+1}\right)$ for $j \in[0, \alpha]$,

where $\theta_{0} \equiv 0$ and $\theta_{\alpha+1} \equiv 2 \pi$.

$\left(A_{4}\right)$ For $j \in[0, \alpha]$,

$$
g\left(\theta_{j}-0\right)=g\left(\theta_{j}+0\right) \text { if } g \equiv x+\pi \text { on }\left(\theta_{j-1}, \theta_{j}^{(g)}\right)
$$

and

$$
\frac{g\left(\theta_{j}-0\right)+g\left(\theta_{j}+0\right)}{2}=\theta_{j} \text { if otherwise. }
$$

Since

$$
\frac{\partial g(\theta)}{\partial \theta} \sin (g(\theta)-\theta)=0
$$

and

$$
\frac{\partial}{\partial \theta}(\cos (g(\theta)-\theta))=\sin (g(\theta)-\theta)
$$

we are able to prove

Lemma 2.6. Following two statements are equivalent.

(a) $\nabla u \in K_{f}$.

(b) There exists $g \in Y$ such that $\nabla u(x)=(\cos g(\arg x), \sin g(\arg x))$ a.e. $x \in B_{1}$.

If a cluster has a wall $l(\theta)$ then the argument function $g$ of function $\nabla u$ is not smooth at $\theta$. By Lemma 2.6 we see that the graph of $g$ has symmetric jump with respect to $\Theta=\theta$ if $g$ jumps at $\theta$. See Figure 2 .

We shall define a $q$-fold energy $E_{f}^{q}$ for $q>0$.

Definition 2.7. $\quad$ For a given $q>0$ we define the $q$-fold energy $E_{f}^{q}$ on $K_{f}$ by

$$
E_{f}^{q}(\nabla u) \equiv \int_{\cup l(\theta)}|[\nabla u](x)|^{q} d \mathcal{S}
$$




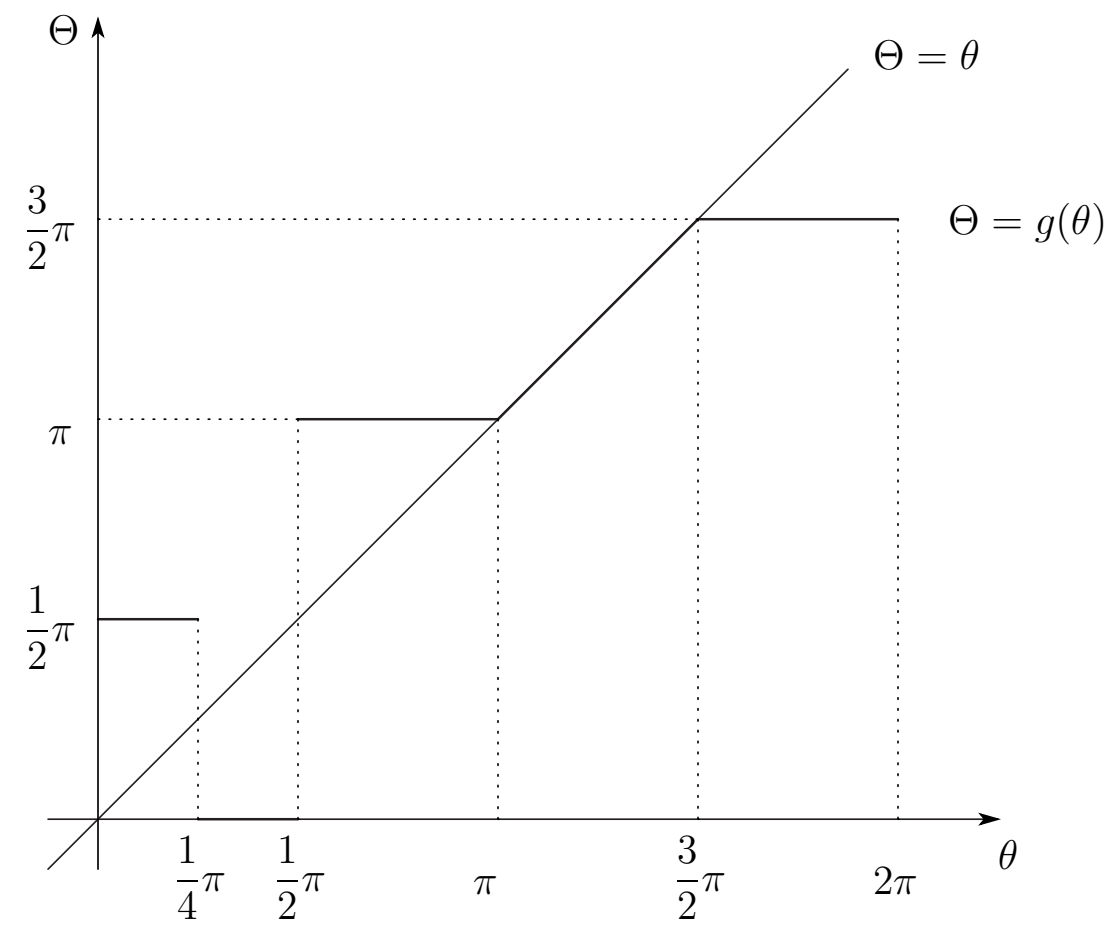

for $\nabla u \in K_{f}$, where $[\nabla u](x)$ denotes the jump of $\nabla u$ at $x=(r \cos \theta, r \sin \theta)$ i.e., Figure 2. The graph of the argument function $g \in Y$ of the cluster in figure 1

$$
[\nabla u](x)=\nabla u(x+0 e)-\nabla u(x-0 e)
$$

with $e=(-\sin \theta, \cos \theta)$. In fact

$$
|[\nabla u]|(x)=2|\sin (g(\theta-0)-\theta)-\theta| .
$$

Next lemma asserts that this fold energy $E_{f}^{q}$ is $L^{1}$-lower semi-continuous on $K_{f}$.

Lemma 2.8. For any cluster $\nabla u \in K_{f}$ and for any positive number $\varepsilon>0$ there exists $\delta_{*}>0$ such that

$$
E_{f}^{q}\left(\nabla u^{\prime}\right)+\varepsilon>E_{f}^{q}(\nabla u)
$$

for any $\nabla u^{\prime} \in K_{f}$ with $\left\|\nabla u-\nabla u^{\prime}\right\|_{L^{1}}<\delta_{*}$.

Proof. Step 1. For $\varepsilon>0$ we shall determine desired $\delta_{*}>0$.

There exists a function $g$ on $(0,2 \pi]$ such that

$$
g(\arg x)=\arg \nabla u(x) \text { and } \theta-\pi<g(\theta) \leq \theta+\pi .
$$

Without loss of generality it is enough to prove in the case $\nabla u \in K_{f} \backslash K_{f}^{0}$ because $E_{f}^{q}(\nabla u)=0$ if $\nabla u \in K_{f}^{0}$. There exists $\tilde{\alpha} \in \mathbb{N}$ and there exists $\left\{\theta_{k}\right\}_{k=1}^{\tilde{\alpha}} \subset(0,2 \pi]$ such that

$$
\theta_{1}<\theta_{2}<\cdots<\theta_{\tilde{\alpha}} \text { and } \tilde{\Sigma}_{\nabla u}=\bigcup_{k=1}^{\tilde{\alpha}} l\left(\theta_{k}\right) .
$$


By a translation of $\theta$ we may assume that

$$
0<\theta_{1}<\theta_{2}<\cdots<\theta_{\tilde{\alpha}}<2 \pi \text {. }
$$

We set $\theta_{0}=0, \theta_{\tilde{\alpha}+1}=2 \pi$ and

$$
\delta_{0} \equiv \frac{1}{4} \min _{1 \leq k \leq \tilde{\alpha}+1}\left\{\theta_{k}-\theta_{k-1}\right\}
$$

If $\Sigma_{\nabla u}=\emptyset$, i.e. $\nabla u$ has no jump, then $E_{f}^{q}(\nabla u)=0$. So we may assume that $\Sigma_{\nabla u} \neq \emptyset$ where

$$
J_{\nabla u}=\left\{\left.\theta \in \tilde{J}_{\nabla u}\left|\int_{l(\theta)}\right|[\nabla u](x)\right|^{q} d \mathcal{S}>0\right\}
$$

and

$$
\Sigma_{\nabla u}=\bigcup_{\theta \in J_{\nabla u}} l(\theta)
$$

There exists $\alpha \in \mathbb{N}$ and $\left\{\theta_{k(j)}\right\}_{j=1}^{\alpha} \subset\left\{\theta_{k}\right\}_{k=1}^{\tilde{\alpha}}$ such that

$$
\theta_{k(1)}<\theta_{k(2)}<\cdots<\theta_{k(\alpha)} \text { and } \Sigma_{\nabla u}=\bigcup_{j=1}^{\alpha} l\left(\theta_{k(j)}\right) .
$$

Since $y=2^{q} \sin ^{q} X$ is continuous on $\mathbb{R}$, for any $\varepsilon>0$ and for any $X \in \mathbb{R}$ there exists $\delta(X, \varepsilon)>0$ such that $\left|2^{q} \sin ^{q} X-2^{q} \sin ^{q} X^{\prime}\right|<\varepsilon$ if $\left|X-X^{\prime}\right|<\delta(X, \varepsilon)$. We set

$$
\begin{aligned}
\delta_{1} \equiv \frac{1}{4} \min _{1 \leq j \leq \alpha}\left\{\left(\theta_{k(j)}+\pi\right)-\arg \nabla u\left(\theta_{k(j)}\right),\left|\arg \nabla u\left(\theta_{k(j)}\right)-\theta_{k(j)}\right|\right. & \\
& \left., \arg \nabla u\left(\theta_{k(j)}\right)-\left(\theta_{k(j)}-\pi\right), \delta_{0}, \delta\left(\left|\arg \nabla u\left(\theta_{k(j)}\right)-\theta_{k(j)}\right|, \frac{\varepsilon}{\alpha}\right)\right\} .
\end{aligned}
$$

It is clear that $\delta_{2} \equiv \sin \delta_{1}>0$ because $0<\delta_{1} \leq \pi / 4$. We set

$$
\delta_{*} \equiv \frac{1}{2} \delta_{1} \delta_{2}
$$

Step 2. We assume that a finite wall cluster $\nabla u^{\prime}$ satisfies $\left\|\nabla u-\nabla u^{\prime}\right\|_{L^{1}}<\delta_{*}$.

We will show that a cluster $\nabla u^{\prime}$ has a wall $l\left(\theta_{j}^{\prime}\right)$ near a wall $l\left(\theta_{k(j)}\right)$ of $\nabla u$.

For any $j \in\{1,2, \cdots, \alpha\}$ there exists $\xi_{j} \in\left\{x \in \Omega_{0} \mid \theta_{k(j)}-\delta_{1}<\arg x<\theta_{k(j)}\right\}$ such that $\left|\nabla u^{\prime}\left(\xi_{j}\right)-\nabla u\left(\xi_{j}\right)\right|<\delta_{2}$, because $\left\|\nabla u^{\prime}-\nabla u\right\|_{L^{1}}<\delta_{*}$. We note that

$$
\nabla u \equiv \nabla u\left(\xi_{j}\right) \text { on }\left[\arg \xi_{j}, \theta_{k(j)}\right]
$$

because $l\left(\theta_{k(j)}\right) \subset \Sigma_{\nabla u}$ and $\arg \xi_{j} \in\left(\theta_{k(j)}-\delta_{0}, \theta_{k(j)}\right)$. Similarly for any $j \in\{1,2, \cdots, \alpha\}$ there exists $\eta_{j} \in\left\{x \in \Omega_{0} \mid \theta_{k(j)}<\arg x<\theta_{k(j)}+\delta_{1}\right\}$ such that $\left|\nabla u^{\prime}\left(\eta_{j}\right)-\nabla u\left(\eta_{j}\right)\right|<$ $\delta_{2}$, and we obtain that

$$
\nabla u \equiv \nabla u\left(\eta_{j}\right) \text { on }\left(\theta_{k(j)}, \arg \eta_{j}\right]
$$


We will argue that

$$
\left|\nabla u\left(\xi_{j}\right)-\nabla u\left(\eta_{j}\right)\right| \geq 2 \sin 2 \delta_{1}
$$

Since

$2 \delta_{1} \leq \min \left\{\left(\theta_{k(j)}+\pi\right)-\arg \nabla u\left(\theta_{k(j)}\right),\left|\arg \nabla u\left(\theta_{k(j)}\right)-\theta_{k(j)}\right|, \arg \nabla u\left(\theta_{k(j)}\right)-\left(\theta_{k(j)}-\pi\right)\right\} \leq \pi / 2$, we see

$$
\sin \left|g\left(\arg \theta_{k(j)}\right)-\theta_{k(j)}\right| \geq \sin 2 \delta_{1} .
$$

Since $l\left(\theta_{k(j)}\right) \subset \Sigma_{\nabla u},(1)$ and (2), we have

$$
\begin{aligned}
\left|\nabla u\left(\xi_{j}\right)-\nabla u\left(\eta_{j}\right)\right| & =2 \sin \frac{\left|\arg \nabla u\left(\xi_{j}\right)-\arg \nabla u\left(\eta_{j}\right)\right|}{2} \\
& =2 \sin \left|\arg \nabla u\left(\theta_{k(j)}\right)-\theta_{k(j)}\right| \\
& \geq 2 \sin 2 \delta_{1} .
\end{aligned}
$$

From the discussion above, we can easily check that

$$
\nabla u^{\prime} \not \equiv \nabla u^{\prime}\left(\xi_{j}\right) \text { on }\left\{x \in \Omega_{0} \mid \arg \xi_{j} \leq \arg x \leq \arg \eta_{j}\right\}
$$

So there exists $\theta_{j}^{\prime} \in\left[\arg \xi_{j}, \arg \eta_{j}\right]$ such that $l\left(\theta_{j}^{\prime}\right) \subset \tilde{\Sigma}_{\nabla u^{\prime}}$. Without loss of generality we may assume that

$$
\theta_{j}^{\prime}=\min \left\{\theta_{j}^{\prime} \in\left[\arg \xi_{j}, \arg \eta_{j}\right] \mid l\left(\theta_{j}^{\prime}\right) \subset \tilde{\Sigma}_{\nabla u^{\prime}}\right\}
$$

Let us check that

$$
g^{\prime}\left(\theta_{j}^{\prime}\right)=g^{\prime}\left(\arg \xi_{j}\right) .
$$

where $g^{\prime}$ is the argument function of $\nabla u^{\prime}$ satisfying $\theta-\pi<g^{\prime}(\theta) \leq \theta+\pi$.

Since

$$
\frac{\left|g^{\prime}\left(\arg \xi_{j}\right)-g\left(\arg \xi_{j}\right)\right|}{2}<\pi-\frac{3}{2} \delta_{1}
$$

and

$$
2 \sin \frac{\left|g^{\prime}\left(\arg \xi_{j}\right)-g\left(\arg \xi_{j}\right)\right|}{2}=\left|\nabla u^{\prime}\left(\xi_{j}\right)-\nabla u\left(\xi_{j}\right)\right|<\delta_{2}<2 \sin \delta_{1},
$$

we find that

$$
\left|g^{\prime}\left(\arg \xi_{j}\right)-g\left(\arg \xi_{j}\right)\right|<2 \delta_{1}
$$

So we can get $g^{\prime}\left(\arg \xi_{j}\right) \neq \arg \xi_{j}$ because

$$
\begin{aligned}
\left|g^{\prime}\left(\arg \xi_{j}\right)-\arg \xi_{j}\right| & \geq\left|g\left(\arg \xi_{j}\right)-\arg \xi_{j}\right|-\left|g\left(\arg \xi_{j}\right)-g^{\prime}\left(\arg \xi_{j}\right)\right| \\
& >4 \delta_{1}-2 \delta_{1}>0 .
\end{aligned}
$$

Similarly we can get $g^{\prime}\left(\arg \xi_{j}\right) \neq \arg \xi_{j} \pm \pi$. Hence $\theta_{j}^{\prime}=\arg \xi_{j}$. Moreover

$$
g^{\prime}\left(\theta_{j}^{\prime}\right)=g^{\prime}\left(\arg \xi_{j}\right)
$$


We can get $g^{\prime}\left(\theta_{j}^{\prime}\right) \neq \theta_{j}^{\prime}$ because

$$
\begin{aligned}
\left|g^{\prime}\left(\theta_{j}^{\prime}\right)-\theta_{j}^{\prime}\right| & =\left|g^{\prime}\left(\arg \xi_{j}\right)-\theta_{j}^{\prime}\right| \\
& \geq\left|g\left(\theta_{k(j)}\right)-\theta_{k(j)}\right|-\left|\theta_{j}^{\prime}-\theta_{k(j)}\right|-\left|g\left(\arg \xi_{j}\right)-g^{\prime}\left(\arg \xi_{j}\right)\right| \\
& >4 \delta_{1}-\delta_{1}-2 \delta_{1}>0
\end{aligned}
$$

by definitions of $\delta_{1}$ and $\theta_{j}^{\prime}$ and by (1), (3) and (4). Similarly we can show that $g^{\prime}\left(\theta_{j}^{\prime}\right) \neq \theta_{j}^{\prime} \pm \pi$.

Since $l\left(\theta_{j}^{\prime}\right) \subset \tilde{\Sigma}_{\nabla u^{\prime}}, g^{\prime}\left(\theta_{j}^{\prime}\right) \neq \theta_{j}^{\prime}$ and $g^{\prime}\left(\theta_{j}^{\prime}\right) \neq \theta_{j}^{\prime} \pm \pi$, we obtain that

$$
\int_{l\left(\theta_{j}^{\prime}\right)}|[\nabla u](x)|^{q} d \mathcal{S}>0
$$

This is equivalent to $l\left(\theta_{j}^{\prime}\right) \subset \Sigma_{\nabla u^{\prime}}$.

Step 3. We will show that the value of the fold energy of $\nabla u^{\prime}$ on $l\left(\theta_{j}^{\prime}\right)$ is nearly equal to one of $\nabla u$ on $l\left(\theta_{k(j)}\right)$. i.e. we will establish that

$$
\left.\left|\int_{l\left(\theta_{k(j)}\right)}\right|[\nabla u]\right|^{q} d \mathcal{H}^{1}-\int_{l\left(\theta_{j}^{\prime}\right)}\left|\left[\nabla u^{\prime}\right]\right|^{q} d \mathcal{H}^{1} \mid<\frac{\varepsilon}{\alpha} .
$$

By definitions of $\delta_{1}$ and $\theta_{j}^{\prime}$ and by (4),

$$
\begin{aligned}
|| g\left(\theta_{k(j)}\right)-\theta_{k(j)}|-| g\left(\theta_{j}^{\prime}\right)-\theta_{j}^{\prime}|| & \leq\left|g\left(\theta_{k(j)}\right)-g\left(\theta_{j}^{\prime}\right)\right|+\left|\theta_{k(j)}-\theta_{j}^{\prime}\right| \\
& <2 \delta_{1}+\delta_{1}<\delta\left(\left|g\left(\theta_{k(j)}\right)-\theta_{k(j)}\right|, \frac{\varepsilon}{\alpha}\right) .
\end{aligned}
$$

Hence

$$
\begin{aligned}
\left.\left|\int_{l\left(\theta_{k(j)}\right)}\right|[\nabla u]\right|^{q} d \mathcal{H}^{1}-\int_{l\left(\theta_{j}^{\prime}\right)}\left|\left[\nabla u^{\prime}\right]\right|^{q} d \mathcal{H}^{1} \mid & =\left|2^{q} \sin ^{q}\right| g\left(\theta_{k(j)}\right)-\theta_{k(j)}\left|-2^{q} \sin ^{q}\right| g\left(\theta_{j}^{\prime}\right)-\theta_{j}^{\prime}|| \\
& <\frac{\varepsilon}{\alpha}
\end{aligned}
$$

using the definition of $\delta\left(\left|g\left(\theta_{k(j)}\right)-\theta_{k(j)}\right|, \varepsilon / \alpha\right)$.

Step 4. For any positive number $\varepsilon>0$ there exists $\delta_{*}>0$ such that

$$
E_{f}^{q}\left(\nabla u^{\prime}\right)+\varepsilon>E_{f}^{q}(\nabla u)
$$

for any $\nabla u^{\prime} \in K_{f}$ with $\left\|\nabla u-\nabla u^{\prime}\right\|_{L^{1}}<\delta_{*}$.

Because

$$
\begin{aligned}
E_{f}^{q}\left(\nabla u^{\prime}\right)+\varepsilon & \geq \sum_{j=1}^{\alpha} \int_{l\left(\theta_{j}^{\prime}\right)}\left|\left[\nabla u^{\prime}\right]\right|^{q} d \mathcal{H}^{1}+\varepsilon \\
& >\sum_{j=1}^{\alpha}\left(\int_{l\left(\theta_{k(j)}\right)}|[\nabla u]|^{q} d \mathcal{H}^{1}-\frac{\varepsilon}{\alpha}\right)+\varepsilon \\
& =\sum_{k=1}^{\tilde{\alpha}} \int_{l\left(\theta_{k}\right)}|[\nabla u]|^{q} d \mathcal{H}^{1}=E_{f}^{q}(\nabla u) .
\end{aligned}
$$

This completes the proof of Lemma 2.8 . 
2.3. Limit clusters. In this section we shall define a limit cluster and its fold energy, and discuss the stability of limit clusters with respect to its fold energy.

Let

$$
\begin{array}{r}
K_{\infty}^{q} \equiv\left\{\left.\nabla u \in K\right|^{\exists}\left\{\nabla u_{j}\right\}_{j \in \mathbb{N}} \subset K_{f} \text { s.t. } \nabla u_{j} \rightarrow \nabla u \text { in } L^{1}\right. \\
\left.\sup E_{f}^{q}\left(\nabla u_{j}\right)<\infty\right\} .
\end{array}
$$

We call this set $K_{\infty}^{q}$ the set of all limit clusters. We consider the $L^{1}$-lower semicontinuous relaxation $E_{\infty}^{q}$ of $E_{f}^{q}$ on $K_{\infty}^{q}$. In fact, for a limit cluster $\nabla u \in K_{\infty}^{q}$, the fold energy of $\nabla u$ is defined by

$$
\begin{array}{r}
E_{\infty}^{q}(\nabla u)=\inf \left\{\underline{\lim } E_{f}^{q}\left(\nabla u_{j}\right) \mid\left\{\nabla u_{j}\right\}_{j \in \mathbb{N}} \subset K_{f} \text { with } \nabla u_{j} \rightarrow \nabla u \text { in } L^{1}\right. \\
\left.E_{f}^{q}\left(\nabla u_{j}\right)<\infty\right\} .
\end{array}
$$

We are able to prove

$$
E_{\infty}^{q}=E_{f}^{q} \text { on } K_{f}
$$

by similar way to prove Lemma 2.8 .

\section{Compactness theorem}

3.1. Compactness theorem on the set of limit clusters. In this section we shall give compactness theorem which is one of our main results. Note that this theorem satisfies for all $q>0$.

Theorem 3. Assume that $\left\{\nabla u_{j}\right\}_{j \in \mathbb{N}} \subset K_{\infty}^{q}$ and that $E_{\infty}^{q}\left(\nabla u_{j}\right)$ is bounded in j. Then there exists a subsequence $\left\{\nabla u_{j(k)}\right\}_{k \in \mathbb{N}}$ of $\left\{\nabla u_{j}\right\}_{j \in \mathbb{N}}$ and there exists a $\nabla u_{0} \in K_{\infty}^{q}$ such that $\nabla u_{j(k)}$ converges to $\nabla u_{0}$ in $L^{1}\left(B_{1}\right)$.

If we admit lemma 3.1, then it is easy to prove theorem 3 by using diagonal method.

Lemma 3.1. Assume that $\left\{\nabla u_{j}\right\}_{j \in \mathbb{N}} \subset K_{f}$ and that $E_{f}^{q}\left(\nabla u_{j}\right)$ is bounded in $j$. Then there exists a subsequence $\left\{\nabla u_{j(k)}\right\}_{k \in \mathbb{N}}$ of $\left\{\nabla u_{j}\right\}_{j \in \mathbb{N}}$ and there exists $\nabla u_{0} \in$ $K_{\infty}^{q}$ such that $\nabla u_{j(k)}$ converges to $\nabla u_{0}$ in $L^{1}\left(B_{1}\right)$.

Proof. The key point in this proof is to pay attention to properties of argument of finite wall clusters.

Step 1. We will introduce a continuous function $h_{j}$ which are made from the argument function $g_{j}$ of $\nabla u_{j}$.

We denote the argument function of $\nabla u_{j}$ satisfying $g_{j}(\arg x)=\arg \nabla u_{j}(x)$ for $x \in B_{1}$ 
by $g_{j}$. We set

$h_{j}(\theta) \equiv\left\{\begin{array}{l}g_{j}(\theta) \text { if } g_{j}(\theta) \geq \theta \\ 2 \theta-g_{j}(\theta) \text { if } g_{j}(\theta)<\theta,\end{array}\right.$

then $h_{j}$ is continuous function. Moreover, $\left\{h_{j}\right\}_{j \in \mathbb{N}}$ is uniformly bounded and equicon-

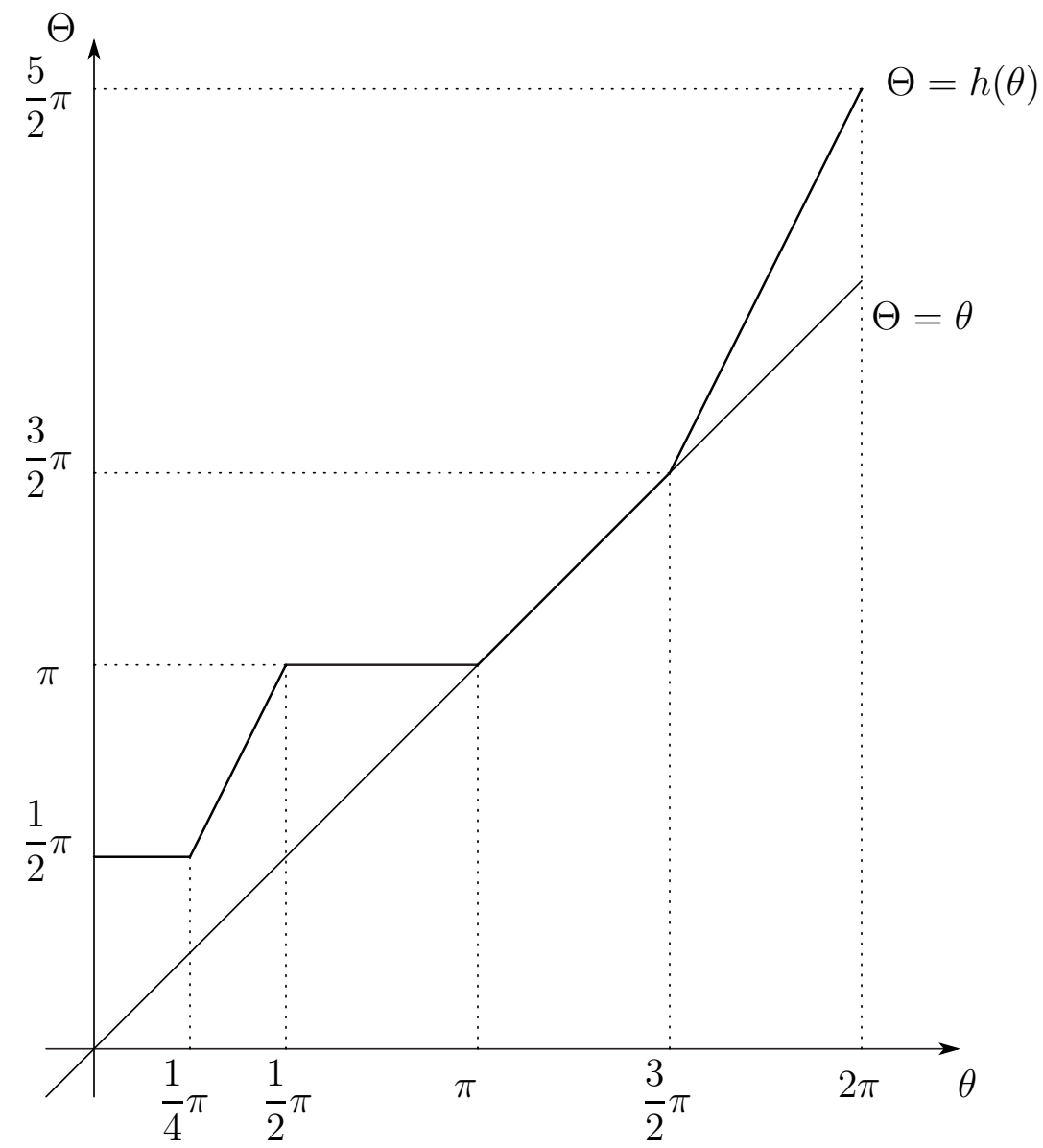

Figure 3. The graph of $h$ of $g$ in figure 1.

tinuous because of lemma 2.6. By Ascoli-Arzelá Theorem, there exists $\left\{h_{j(k)}\right\}_{k \in \mathbb{N}} \subset$ $\left\{h_{j}\right\}_{j \in \mathbb{N}}$ and there exists a continuous function $h_{0}$ such that $h_{j(k)}$ uniformly continuous to $h_{0}$.

Step 2. We will show properties of such $h_{0}$ which are $h_{0}$ is a piecewise smooth function on certain domain and $h^{\prime}=0$ or $h^{\prime}=2$.

For any $n \in \mathbb{N}$ we set

$$
D_{n} \equiv\left\{\left(x_{1}, x_{2}\right) \in \mathbb{R}^{2} \mid x_{1} \in[0,2 \pi], x_{1}+\frac{1}{n}<x_{2}<x_{1}+\pi-\frac{1}{n}\right\}
$$


and

$$
U_{n} \equiv\left\{\theta \in(0,2 \pi) \mid\left(\theta, h_{0}(\theta)\right) \in D_{n}\right\} .
$$

Since $U_{n}$ is open, there exists a sequence $\left\{I_{t}^{(n)}\right\}_{j \in \mathbb{N}}$ of open interval sets such that

$$
\bigcup_{t=1}^{\infty} I_{t}^{(n)}=U_{n}
$$

Notice that $h_{j(k)}$ is piecewise smooth function on $I_{t}^{(n)}$ and $h_{j(k)}^{\prime} \equiv 0$ or $h_{j(k)}^{\prime} \equiv 2$. Since the energy bounded assumption $\sup _{j \in \mathbb{N}} E_{\infty}^{q}\left(\nabla u_{j}\right)<\infty$ and since $h_{j(k)}$ converges to $h_{0}, h_{0}$ is piecewise smooth function on $I_{t}^{(n)}$, too. Moreover $h_{0}^{\prime} \equiv 0$ or $h_{0}^{\prime} \equiv 2$. So $h_{0}$ is piecewise smooth function on $U_{n}$ and $h_{0}^{\prime} \equiv 0$ or $h_{0}^{\prime} \equiv 2$.

Step 3. We will introduce $\nabla u_{0}$ which satisfies $\nabla u_{j}$ tending to $\nabla u_{0}$ in $L^{1}\left(B_{1}\right)$.

We set

and

$$
g_{0}(\theta) \equiv\left\{\begin{array}{l}
h_{0}(\theta) \text { if } \theta \in \bigcup_{t=1}^{\infty} U_{n} \text { and } h_{0}^{\prime}(\theta) \equiv 0 \\
2 \theta-h_{0}(\theta) \text { if } \theta \in \bigcup_{t=1}^{\infty} U_{n} \text { and } h_{0}^{\prime}(\theta) \equiv 2 \\
h_{0}(\theta) \text { if } \theta \notin \bigcup_{t=1}^{\infty} U_{n}
\end{array}\right.
$$

$$
\nabla u_{0} \equiv\left(\cos g_{0}(\arg x), \sin g_{0}(\arg x)\right) .
$$

Then there exists $\left\{\nabla u_{j(k)}\right\}_{k \in \mathbb{N}} \subset\left\{\nabla u_{j}\right\}_{j \in \mathbb{N}}$ such that

$$
\nabla u_{j(k)} \rightarrow \nabla u_{0} \text { a.e. } x \in B_{1}
$$

because $h_{j(k)}$ converges to $h_{0}$. So by bounded convergence theorem, we see that

$$
\nabla u_{j(k)} \rightarrow \nabla u_{0} \text { in } L^{1} .
$$

3.3. Comments for Compactness theorem. In Lemma 3.2 if $q_{0}=r=1$ then $\nabla u_{0} \in K_{\infty}^{q}$ belongs to Aviles-Giga space but $\nabla u_{0}$ is not a function of bounded variation [1]. In [1], Ambrosio, DeLellis and Mantegazza suggest one example which belongs to Aviles-Giga space and which is not a function of bounded variation. Our example is graphically easier to understand than theirs.

Lemma 3.2. Assume that $q_{0} \geq 1 / 2$ and assume that $q>q_{0}$ and $r \leq q_{0}$. Then there exists $\left\{\nabla u_{j}\right\}_{j \in \mathbb{N}} \subset K_{f}^{q}$ with $E_{f}^{q}\left(\nabla u_{j}\right)$ is bounded in $j$ and there exists $\nabla u_{0} \in K_{\infty}^{q}$ such that $\nabla u_{j} \rightarrow \nabla u_{0}$ in $L^{1}\left(\Omega_{0}\right)$ and $\sup _{j \in \mathbb{N}} E_{f}^{r}\left(\nabla u_{j}\right)=\infty$. 
Proof. We will suggest a example of $\nabla u_{j}$ and $\nabla u_{0}$ as required.

We set

$$
A(l) \equiv \sum_{k=1}^{l-1}\left\{\frac{1}{k^{2}}+\left(\frac{1}{k}\right)^{\frac{1}{q_{0}}}-\left(\frac{1}{k+1}\right)^{\frac{1}{q_{0}}}\right\} .
$$

For $j \geq 2$ let

$$
\begin{aligned}
& \left(\begin{array}{ll}
-1 & \text { if } \theta \in\left[0, \frac{1}{2}\right),
\end{array}\right. \\
& 2 \quad \text { if } \theta \in\left[\frac{1}{2}, 2-\left(\frac{1}{2}\right)^{q}\right) \text {, } \\
& A(l)-\left(\frac{1}{l}\right)^{\frac{1}{q_{0}}} \quad \text { if } \theta \in\left[A(l), A(l)+\frac{1}{2 l^{2}}\right), \\
& g_{j}(\theta) \equiv\left\{A(l+1)+\left(\frac{1}{l+1}\right)^{\frac{1}{q_{0}}} \quad \text { if } \theta \in\left[A(l)+\frac{1}{2 l^{2}}, A(l+1)\right)\right. \text {, } \\
& l=2,3, \cdots, j, \\
& A(j+1)+\left(\frac{1}{j+1}\right)^{\frac{1}{q_{0}}} \quad \text { if } \theta \in\left[A(j+1), A(j+1)+\left(\frac{1}{j+1}\right)^{\frac{1}{q_{0}}}\right), \\
& \theta \quad \text { if } \theta \in\left[A(j+1)+\left(\frac{1}{j+1}\right)^{\frac{1}{q_{0}}}, 2 \pi-1\right), \\
& 2 \pi-1 \quad \text { if } \theta \in[2 \pi-1,2 \pi], \\
& \left(\begin{array}{ll}
-1 & \text { if } \theta \in\left[0, \frac{1}{2}\right),
\end{array}\right. \\
& 2 \quad \text { if } \theta \in\left[\frac{1}{2}, 2-\left(\frac{1}{2}\right)^{q}\right) \text {, } \\
& A(l)-\left(\frac{1}{l}\right)^{\frac{1}{q_{0}}} \quad \text { if } \theta \in\left[A(l), A(l)+\frac{1}{2 l^{2}}\right), \\
& g_{0}(\theta) \equiv\left\{\begin{array}{l}
A(l+1)+\left(\frac{1}{l+1}\right)^{\frac{1}{q_{0}}} \quad \text { if } \theta \in\left[A(l)+\frac{1}{2 l^{2}}, A(l+1)\right),
\end{array}\right. \\
& l \leq 2 \text {, } \\
& \text { if } \theta \in\left[\sum_{k=1}^{\infty} \frac{1}{k^{2}}+1,2 \pi-1\right) \text {, } \\
& \text { if } \theta \in[2 \pi-1,2 \pi] \text {. }
\end{aligned}
$$

We set

$$
\nabla u_{j}(x) \equiv\left(\cos g_{j}(\arg x), \sin g_{j}(\arg x)\right) \in K_{f},
$$




$$
\nabla u_{0}(x) \equiv\left(\cos g_{0}(\arg x), \sin g_{0}(\arg x)\right) .
$$

Then we easily check that

$$
\nabla u_{j} \rightarrow \nabla u_{0} \text { in } L^{1} .
$$

If $q>q_{0} \geq 1 / 2$, since

$$
E_{f}^{q}\left(\nabla u_{j}\right) \leq 2^{q} \sum_{k=1}^{j}\left(\frac{1}{k}\right)^{2 q}+2^{q}(q+1) \sup _{0 \leq l \leq q}\left(\begin{array}{l}
q \\
l
\end{array}\right) \sum_{k=1}^{j}\left(\frac{1}{k}\right)^{\frac{q}{q_{0}}}
$$

we obtain

$$
\sup _{j \in \mathbb{N}} E_{f}^{q}\left(g_{j}\right)<\infty
$$

If $r \leq q_{0}$, since

$$
E_{f}^{q}\left(\nabla u_{j}\right) \geq 2^{q} \sum_{k=1}^{j}\left(\frac{1}{k}\right)^{\frac{r}{q_{0}}}
$$

we get

$$
\sup _{j \in \mathbb{N}} E_{f}^{q}\left(g_{j}\right)=\infty
$$

\section{Remark on $L^{1}$-lower semi-continuity theorem}

4.1. $L^{1}$-lower semi-continuity theorem on the set of limit clusters. By the definition of limit clusters it is clear that the fold energy $E_{\infty}^{q}$ is $L^{1}$-lower semicontinuous on the set of limit clusters. Moreover we prove that the fold energy $E_{\infty}^{q}$ is exactly semi-continuous in this section.

Lemma 4.1. The fold energy $E_{\infty}^{q}$ is lower semicontinuous but not continuous on $K_{\infty}^{q}$

Proof. It is enough to prove that the fold energy $E_{f}^{q}$ is not continuous on $K_{f}$. We will suggest a finite wall cluster where the fold energy $E_{f}^{q}$ is not continuous. We set 


$$
g_{j}(\theta) \equiv \begin{cases}\frac{1}{2} \pi & \text { on } \quad\left[0, \frac{1}{4} \pi\right], \\ 0 & \text { on }\left[\frac{1}{4} \pi, \frac{1}{4} \pi+\frac{1}{4 j} \pi\right], \\ \frac{1}{2} \pi+\frac{1}{2 j} \pi & \text { on }\left[\frac{1}{4} \pi+\frac{1}{4 j} \pi, \frac{1}{2} \pi+\frac{1}{2 j} \pi\right], \\ \theta & \text { on }\left[\frac{1}{2} \pi+\frac{1}{2 j} \pi, \frac{3}{2} \pi\right], \\ \frac{3}{2} \pi & \text { on }\left[\frac{3}{2} \pi, 2 \pi\right], \\ g_{0}(\theta) \equiv \begin{cases}\frac{1}{2} \pi & \text { on }\left[0, \frac{1}{2} \pi\right], \\ \theta & \text { on }\left[\frac{1}{2} \pi, \frac{3}{2} \pi\right], \\ \frac{3}{2} \pi & \text { on }\left[\frac{3}{2} \pi, 2 \pi\right],\end{cases} \end{cases}
$$

then $g_{j}, g_{0} \in Y$. And we set

$$
\nabla u_{j}(x) \equiv\left(\cos g_{j}(\arg x), \sin g_{j}(\arg x)\right)
$$

and

$$
\nabla u_{0}(x) \equiv\left(\cos g_{0}(\arg x), \sin g_{0}(\arg x)\right)
$$

Then $\nabla u_{j}$ and $\nabla u_{0}$ are finite wall clusters. We can easily check that

$$
\begin{gathered}
\nabla u_{j} \rightarrow \nabla u_{0} \text { in } L^{1}, \\
E_{f}^{q}\left(\nabla u_{0}\right)=2, \\
E_{f}^{q}\left(\nabla u_{j}\right)=2\left(\frac{\sqrt{2}}{2}\right)^{q}+2+2 \sin ^{q}\left|g_{j}\left(\frac{1}{4} \pi+\frac{1}{4 j} \pi\right)-\frac{1}{4} \pi+\frac{1}{4 j} \pi\right| .
\end{gathered}
$$

Then for any $j$

$$
E_{f}^{q}\left(\nabla u_{0}\right)-E_{f}^{q}\left(\nabla u_{j}\right) \geq 2\left(\frac{\sqrt{2}}{2}\right)^{q}>0
$$

This completes the proof of theorem 4.1. 


\section{Global minimizers and examples of local mini- mizer}

In this chapter we present global minimizers and examples of local minimizers.

5.1. Global minimizers. Global minimizers are sorted into three patterns as follows.

Theorem 5.1. Following two statements are equivalent.

(a) $\nabla u \in K_{\infty}^{q}$ is a global minimizer on $K_{\infty}^{q}$ of $E_{\infty}^{q}$.

(b) $\nabla u \in K_{\infty}^{q}$ is either $\arg \nabla u(x)=\arg (x)$ or $\arg \nabla u(x)=\arg (-x)$ or $\arg \nabla u \equiv C$.

Proof. If (b) holds then we can get (a) because a fold energy of any clusters with (b) is zero. On the other side if (a) holds then a global minimizer $\nabla u$ has zero energy. So $\nabla u$ has no energy on all walls of $\nabla u$. Therefore $\nabla u$ is a finite wall cluster and by the feature of finite wall clusters $\nabla u$ satisfies (b).

5.2. Examples of local minimizer. We consider a typical series of configurations which is expected to be stationary for $E_{\infty}^{q}$.

Definition 5.2. For given $\left\{\theta_{j}^{*}\right\}_{j=1}^{2 n} \subset(0,2 \pi]$ satisfying

$$
0<\theta_{1}^{*}<\theta_{2}^{*}<\cdots<\theta_{2 n}^{*} \leq 2 \pi
$$

and

$$
\theta_{j+1}^{*}=\theta_{j}^{*}+\frac{1}{n} \pi \text { for }{ }^{\forall} j=1,2, \cdots, 2 n-1
$$

we define $\nabla u_{*}^{(n)} \in K_{f}^{q}$ as follows.

$\nabla u_{*}^{(n)} \in K_{f}^{q}$ has $2 n$ walls $\left\{l\left(\theta_{j}^{*}\right)\right\}_{j=1}^{2 n}$ and satisfies for all $x \in \Omega_{0}$ with $\arg x \in\left(\theta_{1}^{*}, \theta_{2}^{*}\right)$

$$
\nabla u_{*}^{(n)}(x)=\left(\cos \left(\theta_{1}^{*}+\frac{n+1}{2 n}\right), \sin \left(\theta_{1}^{*}+\frac{n+1}{2 n}\right)\right)
$$

or

$$
\nabla u_{*}^{(n)}(x)=\left(\cos \left(\theta_{1}^{*}-\frac{n+1}{2 n}\right), \sin \left(\theta_{1}^{*}-\frac{n+1}{2 n}\right)\right) .
$$

We denote the set of such $\nabla u_{*}^{(n)}$ 's by $Z_{2 n}$. 


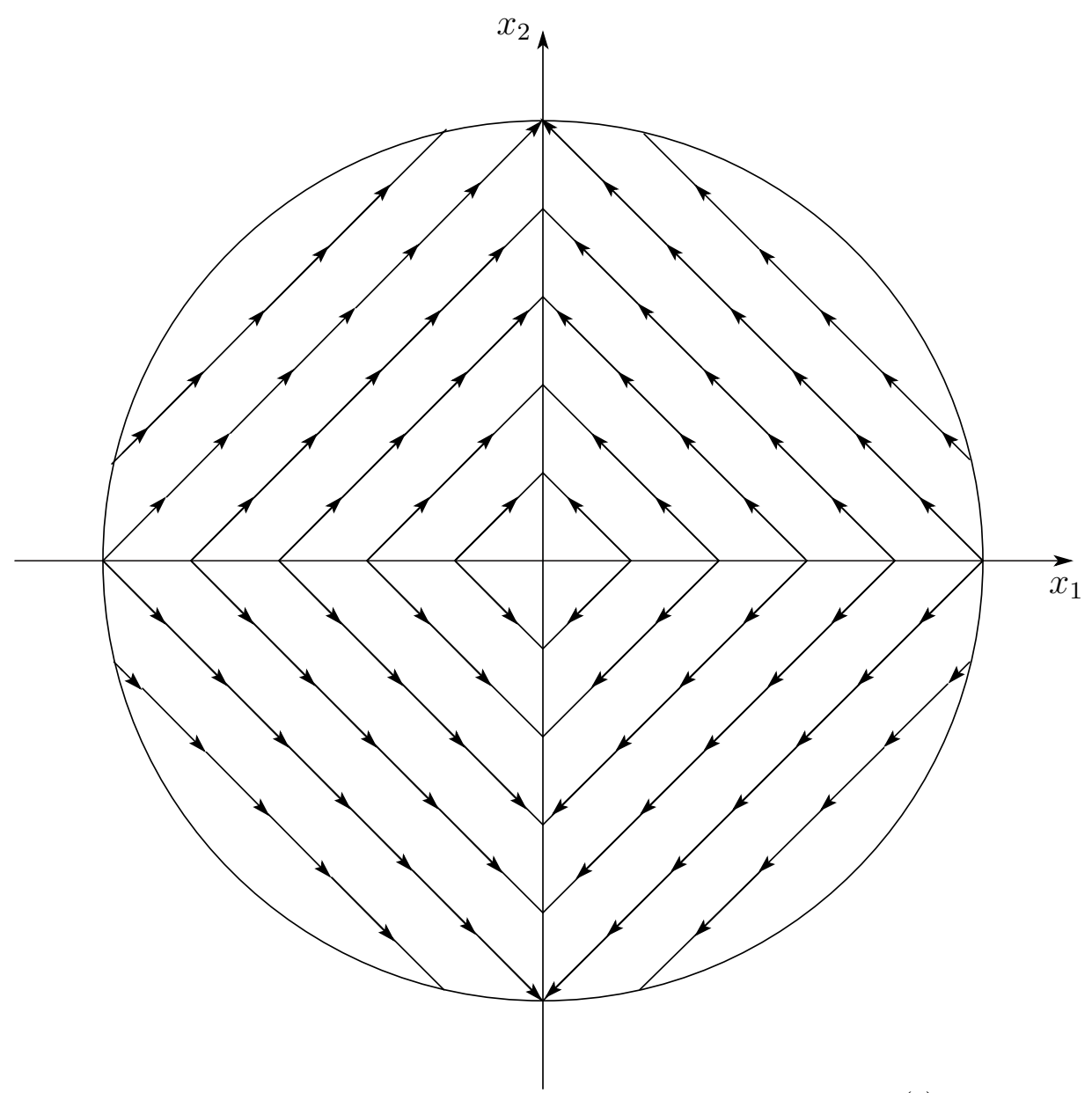

Figure 4. The gradient field of $\nabla u_{*}^{(2)}$

All elements of $Z_{2 n}$ are local minimizers if $q \sin ^{2} \frac{\pi}{2 n}>1$. If $q>0$ is large then $\nabla u_{*}^{(n)} \in Z_{n}$ tends to be a local minimizer and if $n \in \mathbb{N}$ is large then $\nabla u_{*}^{(n)} \in Z_{n}$ tends to be a local minimizer.

Theorem 5.3. Assume that $q>0$ and $n \in \mathbb{N} \backslash\{1\}$ satisfy $q \sin ^{2} \frac{\pi}{2 n}>1$ and that $\nabla u_{*}^{(n)} \in Z_{2 n}$. Then there exists $\varepsilon_{0}>0$ such that $E_{\infty}^{q}\left(\nabla u_{*}^{(n)}\right)<E_{\infty}^{q}(\nabla u)$ for any $\nabla u \in K_{\infty}^{q}$ with $\left\|\nabla u_{*}^{(n)}-\nabla u\right\|_{L^{1}}<\varepsilon_{0}$. Moreover, if $q>0$ and $n \in \mathbb{N} \backslash\{1\}$ satisfy $q \sin ^{2} \frac{\pi}{2 n}<1$ then $\nabla u_{*}^{(n)} \in Z_{2 n}$ are not local minimizers.

If we admit lemma 5.4, then it is easy to check theorem 5.4. so it is enough to prove lemma 5.4 .

Lemma 5.4. Assume that $q>0$ and $n \in \mathbb{N} \backslash\{1\}$ satisfy $q \sin ^{2} \frac{\pi}{2 n}>1$ and that $\nabla u_{*}^{(n)} \in Z_{2 n}$. Then there exists $\varepsilon_{0}^{\prime}>0$ such that $E_{f}^{q}\left(\nabla u_{*}^{(n)}\right)<E_{f}^{q}(\nabla u)$ for any $\nabla u \in K_{f}^{q}$ with $\left\|\nabla u_{*}^{(n)}-\nabla u\right\|_{L^{1}}<\varepsilon_{0}^{\prime}$. i.e. $\nabla u_{*}^{(n)} \in Z_{2 n}$ are local minimizers. 
Proof. Step 1. It is enough to check that finite wall clusters $\nabla u_{*}^{(n)}$ are minimizers of fold energy $E_{f}^{q}$ on the set of finite wall clusters $\nabla u^{\prime}$ which have $2 n$ walls $\left\{l\left(\theta_{j}^{\prime}\right)\right\}_{j=1}^{2 n}$ with $\bigcup_{j=1}^{2 n} l\left(\theta_{j}^{\prime}\right)=\Sigma_{\nabla u^{\prime}}=\tilde{\Sigma}_{\nabla u^{\prime}}$ because of next Claim.

Claim . Let $\varepsilon_{* *} \equiv \sin ^{q}\left(\frac{1}{2} \pi+\frac{11}{16 n} \pi\right)$ and let $\delta_{*}=\delta_{*}\left(\varepsilon_{* *}\right)$ as Lemma 2.8. If $\nabla u^{\prime} \in K_{f}$ with $\left\|\nabla u_{*}^{(n)}-\nabla u^{\prime}\right\|_{L^{1}}<\delta_{*}$ has a wall $l\left(\theta_{2 n+1}^{\prime}\right) \subset \Sigma_{\nabla u^{\prime}}$ besides $2 n$ walls $\left\{l\left(\theta_{j}^{\prime}\right)\right\}_{j=1}^{2 n}$, then

$$
E_{f}^{q}\left(\nabla u^{\prime}\right)>E_{f}^{q}\left(\nabla u_{*}^{(n)}\right)+\varepsilon_{* *} .
$$

Proof of Claim. By the definition of $\nabla u_{*}^{(n)}$ and by $\left\|\nabla u_{*}-\nabla u^{\prime}\right\|_{L^{1}}<\delta_{*}$, we are able to check that $l\left(\theta_{2 n+1}^{\prime}\right) \subset \tilde{\Sigma}_{\nabla u^{\prime}}$. Without loss of generality we can assume that $\theta_{1}^{\prime}<\theta_{2 n+1}^{\prime}<\theta_{2}^{\prime}$. Let

$$
\theta_{\max }^{\prime} \equiv \max \left\{\theta^{\prime} \in\left(\theta_{1}^{\prime}, \theta_{2}^{\prime}\right) \mid l\left(\theta^{\prime}\right) \subset \tilde{\Sigma}_{\nabla u^{\prime}} \backslash \bigcup_{j=1}^{2 n} l\left(\theta_{j}^{\prime}\right)\right\}
$$

and let $g^{\prime}$ be a function on $(0,2 \pi]$ satisfying $\theta-\pi<g^{\prime}(\theta)<\theta+\pi$ and $g^{\prime}(\arg x)=$ $\arg \nabla u^{\prime}(x)$ on $\Omega$. Because

$$
0<\frac{1}{2} \pi+\frac{5}{16 n} \pi<\theta_{\max }^{\prime}-g^{\prime}\left(\theta_{\max }^{\prime}+0\right)<\frac{1}{2} \pi+\frac{11}{16 n} \pi<\pi
$$

we can get

$$
\begin{aligned}
\int_{l\left(\theta_{\max }^{\prime}\right)}\left|\left[\nabla u^{\prime}\right]\right|^{q} d \mathcal{H}^{1} & =2 \sin ^{q}\left|g^{\prime}\left(\theta_{\max }^{\prime}+0\right)-\theta_{\max }^{\prime}\right| \\
& >2 \sin ^{q}\left(\frac{1}{2} \pi+\frac{11}{16 n} \pi\right)=2 \varepsilon_{* *} .
\end{aligned}
$$

Consequently

$$
\begin{aligned}
E_{f}^{q}\left(\nabla u^{\prime}\right) & \geq \sum_{k=1}^{2 n} \int_{l\left(\theta_{k}^{\prime}\right)}\left|\left[\nabla u^{\prime}\right]\right|^{q} d \mathcal{H}^{1}+\int_{l\left(\theta_{\max }^{\prime}\right)}\left|\left[\nabla u^{\prime}\right]\right|^{q} d \mathcal{H}^{1} \\
> & \left(E_{f}^{q}\left(\nabla u_{*}^{(n)}\right)-\varepsilon_{* *}\right)+2 \varepsilon_{* *}=E_{f}^{q}\left(\nabla u_{*}^{(n)}\right)+\varepsilon_{* *} .
\end{aligned}
$$

This complete the proof of Claim.

Step 2. By Step 1, for any clusters $\nabla u^{\prime}$ with $\left\|\nabla u_{*}^{(n)}-\nabla u^{\prime}\right\|_{L^{1}}<\delta_{*}$ we will represent the fold energy $E_{f}^{q}$ of $\nabla u^{\prime}$ by $2 n-1$ parameters.

A routine calculation yields

$$
\int_{l\left(\theta_{1}\right)}\left|\left[\nabla u^{\prime}\right]\right|^{q} d \mathcal{H}^{1}=2^{q} \sin ^{q}\left(\alpha-\theta_{1}\right)
$$




$$
\int_{l\left(\theta_{2 n}\right)}\left|\left[\nabla u^{\prime}\right]\right|^{q} d \mathcal{H}^{1}=2^{q} \sin ^{q} \alpha
$$

and for any $k \in[2,2 n-1]$

$$
\int_{l\left(\theta_{k}\right)}\left|\left[\nabla u^{\prime}\right]\right|^{q} d \mathcal{H}^{1}=2^{q} \sin ^{q}(-1)^{k}\left\{(-1)^{k} \alpha+\theta_{k}+2 \sum_{h=1}^{k-1}(-1)^{h} \theta_{k-h}\right\} .
$$

where $g^{\prime}$ is the argument function of $\nabla u^{\prime}, \alpha=g^{\prime}(0+0)$ and $\left\{l\left(\theta_{j}^{\prime}\right)\right\}_{j=1}^{2 n}$ are all walls of $\nabla u^{\prime}$.

The other side $\sum_{l=1}^{2 n-1}(-1)^{l+1} \theta_{2 n-l}=2 \pi$ because

$$
\frac{g\left(\theta_{2 n}-0\right)+g\left(\theta_{1}-0\right)+2 \pi}{2}=2 \pi
$$

So

$$
\int_{l\left(\theta_{2 n-1}\right)}\left|\left[\nabla u^{\prime}\right]\right|^{q} d \mathcal{H}^{1}=2^{q} \sin ^{q}\left(\sum_{h=2}^{2 n-1}(-1)^{h+1} \theta_{2 n-h}-\alpha\right) .
$$

Therefore the fold energy of $\nabla u^{\prime}$ is represented by $2 n-1$ parameters $\alpha, \theta_{1}, \theta_{2}, \cdots, \theta_{2 n-2}$. i.e.

$$
\begin{gathered}
E_{f}^{q}\left(\nabla u^{\prime}\right)=2^{q} \sin ^{q}\left(\alpha-\theta_{1}\right)+2^{q} \sum_{k=2}^{2 n-2} \sin ^{q}(-1)^{k}\left\{(-1)^{k} \alpha+\theta_{k}+2 \sum_{h=1}^{k-1}(-1)^{h} \theta_{k-h}\right\} \\
+2^{q} \sin ^{q}\left(\sum_{k=2}^{2 n-1}(-1)^{h+1} \theta_{2 n-h}-\alpha\right)+2^{q} \sin ^{q} \alpha
\end{gathered}
$$

Step 3. We set

$$
R^{q}\left(\alpha, \theta_{1}, \cdots, \theta_{k}, \cdots, \theta_{2 n-2}\right)=\frac{E_{f}^{q}\left(\nabla u^{\prime}\right)}{2^{q}} \text { for }\left\|\nabla u_{*}^{(n)}-\nabla u^{\prime}\right\|_{L^{1}}<\delta_{*}
$$

and

$$
P=\left(\frac{n+1}{2 n} \pi, \frac{1}{n} \pi, \cdots, \frac{k}{n} \pi, \cdots, \frac{2 n-2}{n} \pi\right) .
$$

A routine calculation yields that

$$
E_{f}^{q}\left(\nabla u_{*}^{(n)}\right)=2^{q} R^{q}(P),
$$

$P$ is a critical point of $R^{q}$ 
and

$$
\operatorname{Hess} R^{q}(P)=q\left\{q\left(\sin \frac{\pi}{2 n}\right)^{2}-1\right\}\left(\cos \frac{\pi}{2 n}\right)^{q-2}\left(a_{i j}\right)_{1 \leq i, j \leq 2 n-1}
$$

where

$$
\begin{gathered}
a_{i j}=a_{j i}, a_{11}=2 n, \\
a_{m m}=8 n-2-4 m \text { if } m \neq 1, \\
a_{1 m}=(-1)^{m+1}(4 n-2 m) \text { if } m \neq 1, \\
a_{t m}=(-1)^{m+t}(-8 n-4 t-1) \text { if } t>m>1 .
\end{gathered}
$$

In this step we will prove that $\operatorname{Hess}^{q}(P)>0$.

We set

$$
D_{k} \equiv\left(a_{i j}\right)_{1 \leq i, j \leq k}
$$

Because if $k$ is odd then

$$
D_{k} \sim\left(\begin{array}{ccccccc}
2 n-(k-1) & 3 & 0 & 0 & 0 & \cdots & 0 \\
3 & 6 & -1 & 0 & 0 & \cdots & 0 \\
0 & -1 & 2 & -1 & 0 & \cdots & 0 \\
0 & 0 & -1 & 2 & -1 & \cdots & 0 \\
0 & 0 & 0 & -1 & 2 & \ddots & 0 \\
\vdots & \vdots & \vdots & \ddots & \ddots & \ddots & -1 \\
0 & 0 & 0 & 0 & 0 & -1 & 2
\end{array}\right)
$$

and because if $k$ is even then

$$
D_{k} \sim\left(\begin{array}{ccccccc}
2 n-k & -2 & 0 & 0 & 0 & \cdots & 0 \\
-2 & 6 & -1 & 0 & 0 & \cdots & 0 \\
0 & -1 & 2 & -1 & 0 & \cdots & 0 \\
0 & 0 & -1 & 2 & -1 & \cdots & 0 \\
0 & 0 & 0 & -1 & 2 & \ddots & 0 \\
\vdots & \vdots & \vdots & \ddots & \ddots & \ddots & -1 \\
0 & 0 & 0 & 0 & 0 & -1 & 2
\end{array}\right)
$$

we are able to get

$$
\operatorname{det} D_{k}= \begin{cases}-5 k^{2}+5+10 n k-8 n>0 & \text { if } k \text { is odd, } \\ -5 k^{2}+4+10 n k-8 n>0 & \text { if } k \text { is even }\end{cases}
$$

where $1 \leq k \leq 2 n-1$. Therefore $\operatorname{Hess} R^{q}(P)>0$ because $\left(a_{i j}\right)_{1 \leq i, j \leq 2 n-1}>0$. This completes the proof of Lemma 5.4. 


\section{References}

[1] L. Ambrosio, C. DeLellis and C. Mantegazza, Line energies for gradient vector fields in the plane, Calc. Var. 9(1999), 327-355

[2] P. Aviles and Y. Giga, The distance function and defect energy. Proc. Roy. Soc. Edinburgh Sect, A, 126(1996), 923-938

[3] P. Aviles and Y. Giga, On lower semicontinuity of a defect energy obtained by a singular perturbation of a Ginzburg-Landau type energy for gradient fields, Proc. Roy. Soc. Edinburgh Sect. A, 129(1999), 1-17

[4] De Lellis, Camillo and Otto, Felix Structure of entropy solutions to the eikonal equation, J. Eur. Math. Soc. (JEMS) 5 (2003), no. 2, 107-145.

[5] A. DeSimone and R. V. Kohn, S. Müller and F. Otto, A compactness result in the gradient theory of phase transitions, Proc. Roy. Soc. Edinburgh Sect. A, $131(2001), 833-844$

[6] P. -E. Jabin, F. Otto and B. Perthame, Line-energy Ginzburg-Landau models: zero-energy states, Ann. Sc. Norm. Super. Pisa Cl. Sci. (5) 1 (2002), no. 1, $187-202$.

[7] W. Jin and R. V. Kohn, Singular perturbation and the energy of folds J. Nonlinear Science. 10 (2000), 355-390.

[8] Hugo A. M. van den Berg, Order in the domain structure in soft-magnetic thinfilm elements, IBM J. Res. Develop. Vol.33 No.5 September (1989), 540-582. 